\title{
Automatische Einkommensteuererstattungen zur Entlastung niedriger Einkommen
}

\author{
Deutschland ist eines von mehr als 30 Ländern weltweit, in denen Beschäftigte von der \\ Pflicht zur Abgabe einer Steuererklärung befreit sind. Diese optionale Veranlagung befreit \\ vom Aufwand einer Steuererklärung, hat aber individuelle und verteilungspolitische Kosten, \\ weil Nichtveranlagte häufig zu viel Lohnsteuer bezahlen. Dieser Übereinbehalt führt zu \\ beträchtlichen Abweichungen der effektiven Steuerlast vom Steuertarif, vor allem in niedrigen \\ Einkommensbereichen und insbesondere bei Beschäftigten mit Bruttoarbeitslöhnen unterhalb \\ des Grundfreibetrags. Insgesamt zahlen nichtveranlagte Steuerzahler:innen deutlich zu \\ viel Einkommensteuer - allein im Jahr 2014 mindestens 949 Mio. Euro. Eine automatische \\ Erstattung dieses Übereinbehalts würde vor allem geringe Einkommen entlasten.
}

\begin{abstract}
In mehr als 30 Ländern weltweit ist es Arbeitnehmer:innen freigestellt, eine Steuererklärung abzugeben (Gale und Holtzblatt, 1997; US Department of the Treasury, 2003). Auch das deutsche Einkommensteuergesetz erlaubt ausschließlich abhängig Beschäftigten, auf die Abgabe einer Steuererklärung zu verzichten, sofern keine weiteren Einkünfte oder Einkommensersatzleistungen (etwa Elterngeld) vorliegen. Sie können freiwillig eine Steuererklärung abgeben, müssen dies aber nicht, da ihre Arbeitgeber:innen automatisch Lohnsteuer für sie abführen. Anhand der Lohn- und Einkommensteuerstatistik 2014, dem aktuellsten Mikrodatensatz zu Steuerpflichtigen in Deutschland, analysieren wir die Effekte dieser Wahlfreiheit.
\end{abstract}

In den vergangenen Jahren haben etwa $60 \%$ der Steuerpflichtigen mit optionaler Veranlagung auf die Abgabe einer Steuererklärung verzichtet, das entspricht circa

(C) Der/die Autor:in 2021. Open Access: Dieser Artikel wird unter der Creative Commons Namensnennung 4.0 International Lizenz veröffentlicht (creativecommons.org/licenses/by/4.0/deed.de).

Open Access wird durch die ZBW - Leibniz-Informationszentrum Wirtschaft gefördert.

Tobias Hauck war bis August 2021 wissenschaftlicher Mitarbeiter an der Ludwig-MaximiliansUniversität München.

Luisa Wallossek ist Doktorandin an der LudwigMaximilians-Universität München.
$30 \%$ aller Steuerpflichtigen in Deutschland. ${ }^{1}$ Unsere Untersuchung zeigt, dass vor allem Steuerzahlende mit geringem Einkommen selten eine Steuererklärung abgeben. Wir zeigen, dass diese Nichtveranlagung dazu führt, dass Steuerzahler:innen erheblich mehr Lohnsteuer zahlen, als es der Einkommensteuertarif vorsieht, indem wir die tarifliche Lohnsteuer berechnen und diese mit der tatsächlich einbehaltenen, effektiven Lohnsteuer vergleichen. Der Vergleich zeigt: Nichtveranlagte Steuerpflichtige haben 2014 insgesamt mindestens 949 Mio. Euro (durchschnittlich 119 Euro pro Kopf) zu viel Lohnsteuer entrichtet, weil der Lohnsteuerabzug zu Übereinbehalten führt. Durch den Verzicht einer Steuererklärung verpassen Steuerzahlende signifikante Rückerstattungen.

Die Abweichung zwischen effektiver und tariflicher Besteuerung ist dabei in den untersten Einkommensbereichen besonders ausgeprägt. Unterhalb des Grundfreibetrags, der 2014 bei alleinstehenden Steuerpflichtigen einem Bruttoarbeitslohn von knapp 11.000 Euro entspricht, ist die tarifliche Lohnsteuer null. Tatsächlich zahlen Nichtveranlagte hier im Schnitt aber $2 \%$ Lohnsteuer. Berücksichtigt man nur diejenigen, die zu viel Lohnsteuer entrichten, beträgt der effektive Durchschnittssteuersatz etwa $5 \%$. Im Ergebnis ist die effektive Besteuerung weniger progressiv, als es der Einkommensteuertarif vorsieht. Wir halten dies, nicht zuletzt aufgrund der höchstrichterlichen Entscheidung zur Steuerfreiheit des Grundfreibetrags, für bedenklich und leiten Handlungsempfehlungen zur Eliminierung dieser Abweichung und Entlastung geringer Einkommen ab.

1 Die aggregierten Daten zur Veranlagung basieren auf der Lohn- und Einkommensteuerstatistik 2007 bis 2016 und wurden auf Anfrage vom Statistischen Bundesamt zur Verfügung gestellt. 


\section{Daten und Methodik}

Um die Auswirkung freiwilliger Veranlagung und der resultierenden Nichtveranlagung auf die effektive Einkommensbesteuerung zu untersuchen, nutzen wir die aktuellste Version der Lohn- und Einkommensteuerstatistik (LEST) für das Jahr 2014 (Forschungsdatenzentrum des Bundes und der Länder, 2020). Der Datensatz ist eine stratifizierte Zufallsstichprobe und beinhaltet neben veranlagten Steuerpflichtigen auch 400.000 Nichtveranlagte. Die Daten für Nichtveranlagte stammen aus den Lohnsteuerjahresbescheinigungen der Arbeitgeber:innen und beinhalten neben demografischen Merkmalen das Bruttoeinkommen sowie die einbehaltene Lohnsteuer, die der effektiven Einkommensteuer entspricht. Für unsere Analyse begrenzen wir den Datensatz auf Individuen, für welche die Abgabe einer Steuererklärung freiwillig ist.

Ausgehend vom Bruttojahreseinkommen können wir auch für Nichtveranlagte die Einkommensteuer nach Tarif berechnen und diese mit der effektiv einbehaltenen Lohnsteuer vergleichen. Wir berechnen das zu versteuernde Einkommen und die entsprechende tarifliche Einkommensteuer auf Basis der Programmablaufpläne des Bundesministeriums für Finanzen. Wir berücksichtigen die Pauschbeträge für Werbungskosten und Sonderausgaben sowie die abzugsfähigen Vorsorgeaufwendungen. Da diese abzugsfähigen Vorsorgeaufwendungen mit der LEST nur für Steuerpflichtige in der gesetzlichen Renten-, Kranken- und Pflegeversicherung eindeutig berechenbar sind, begrenzen wir die Stichprobe weiter auf Angestellte (keine Beamt:innen) mit Bruttojahreslohn unterhalb der Beitragsbemessungsgrenze der Krankenversicherung (48.600 Euro 2014). Zudem nehmen wir für alle Individuen den durchschnittlichen Zusatzbetrag der gesetzlichen Krankenversicherungen an. ${ }^{2}$

Unsere Analyse bezieht sich auf das aktuellste verfügbare Erhebungsjahr mit Daten für 2014 (Forschungsdatenzentrum des Bundes und der Länder, 2020). Wir reproduzieren unsere Ergebnisse für 2010 (Forschungsdatenzentrum des Bundes und der Länder, 2018), das zweitaktuellste Erhebungsjahr. Die Berechnungen für 2010 sind qualitativ gleich und quantitativ ähnlich zu 2014, wenngleich absolut etwas geringer (Hauck und Wallossek, 2021).

2 Durch diese aufgrund der Datenverfügbarkeit notwendigen vereinfachenden Annahmen können kleinere Ungenauigkeiten entstehen. Daher erlauben wir bei der Berechnung des Übereinbehalts eine Toleranz von +/- 5 Euro. Abweichungen vom Steuertarif in diesem Bereich werten wir nicht als eigentliche Abweichung, Abweichungen $>5$ Euro werden als Übereinbehalt gewertet, Observationen mit Abweichungen $<-5$ Euro werden nicht weiter berücksichtigt. In diesen Fällen ist davon auszugehen, dass sich steuerrelevante Merkmale unterjährig geändert haben, was in den jährlichen Daten nicht ersichtlich ist.
Abbildung 1

Nichtveranlagung über die Einkommensverteilung

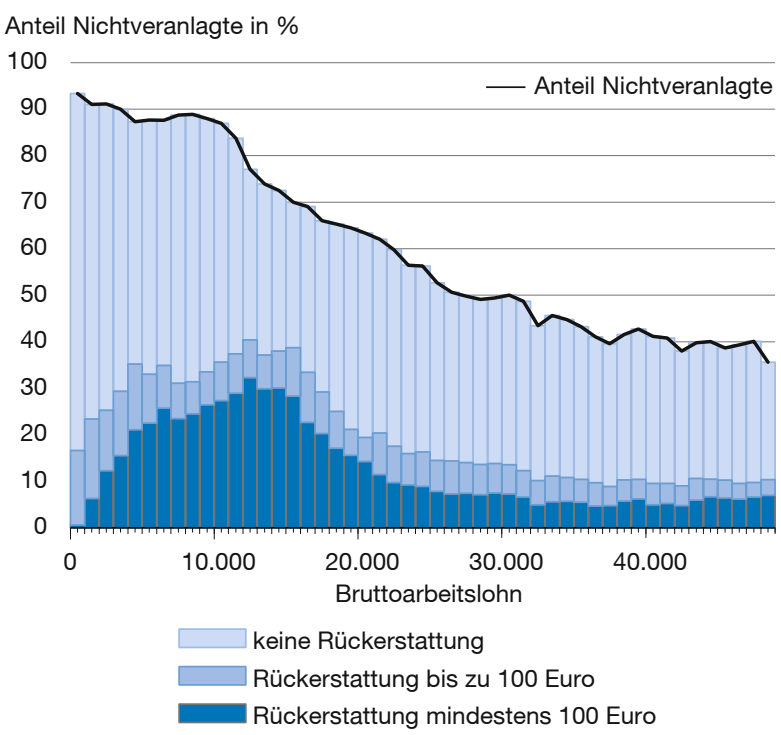

Interpretationshilfe: Von allen Arbeitnehmer:innen mit einem Bruttoarbeitslohn von 10.000 Euro geben $88 \%$ keine Steuererklärung ab (Summe der Balken und Höhe der durchgezogenen Linie). Diese Nichtveranlagten sind in drei Gruppen kategorisiert. Diejenigen, die keine Rückerstattung erwarten können (hellblau, $54 \%$ ), diejenigen mit Rückerstattungen bis zu 100 Euro (mittelblau, $7 \%$ ) und diejenigen mit einer Erstattung von mindestens 100 Euro (dunkelblau, $26 \%$ ).

Quelle: Forschungsdatenzentrum des Bundes und der Länder (2020); eigene Berechnungen.

\section{Ergebnisse}

Nichtveranlagung über die Einkommensverteilung

Abbildung 1 zeigt den Anteil der Nichtveranlagten über das jährliche Bruttoeinkommen für Steuerzahlende mit optionaler Veranlagung (schwarze Linie). Dabei besteht ein klarer negativer Zusammenhang zwischen der Wahrscheinlichkeit, eine Steuererklärung abzugeben, und dem jährlichen Bruttoverdienst. Am unteren Ende der Verteilung, bis zu einem Jahresbruttoeinkommen von etwa 10.000 Euro, geben $90 \%$ keine Steuererklärung ab. Der Anteil sinkt mit steigendem Bruttojahreslohn bis er ein Niveau von etwa $30 \%$ erreicht.

Dass Steuerpflichtige keine Steuererklärung abgeben, impliziert nicht automatisch einen finanziellen Nachteil. Wenn der automatische Lohnsteuereinbehalt gut funktioniert und zusätzlich keine Ausgaben jenseits der Pauschbeträge abgesetzt werden können, führt die Abgabe einer Steuererklärung zu keiner Steuererstattung. Dies könnte potenziell erklären, warum vor allem Geringverdienende keine Steuererklärung abgeben. Wer in einem Angestelltenverhältnis weniger als 11.000 Euro brutto jährlich verdient, sollte laut 
Steuertarif keine Einkommensteuer zahlen, da das korrespondierende zu versteuernde Einkommen innerhalb des Grundfreibetrags liegt (Bundesministerium der Finanzen, 2019). Wenn keine Lohnsteuer einbehalten wird, gibt es folglich keinen Anreiz, eine Steuererklärung abzugeben. Wir zeigen jedoch, dass dies häufig nicht der Fall ist.

Übereinbehalte entstehen, weil Arbeitgeber:innen der monatlichen Lohnsteuer ein hypothetisches Jahreseinkommen zugrunde legen, das nicht notwendigerweise dem tatsächlichen Jahreseinkommen entspricht. Bei Fluktuationen im Einkommen bewirkt die Progressivität des Steuertarifs eine zu hohe Lohnsteuer, die Arbeitgeber:innen nicht immer korrigieren (können). Zudem werden die Pauschbeträge für Werbungskosten und Sonderausgaben nicht vollständig berücksichtigt, wenn Arbeitnehmer:innen nicht das gesamte Jahr beschäftigt sind. Die zugrundeliegenden Mechanismen werden ausführlicher in Hauck und Wallossek (2021) beschrieben.

\section{Steuererstattungspotenzial}

Abbildung 1 dokumentiert zunächst, dass der automatische Lohnsteuereinbehalt für die Mehrheit der Nichtveranlagten gut funktioniert. Bei Abgabe einer Steuererklärung ohne Ausgaben jenseits der Pauschbeträge können sie keine Rückerstattung erwarten (hellblaue Balken). Teilweise gibt es jedoch moderate (bis 100 Euro, mittelblaue Balken) oder starke (100 Euro und mehr, dunkelblaue Balken) Differenzen zwischen einbehaltener und tariflicher Lohnsteuer. Vor allem im unteren Einkommensbereich zahlen Nichtveranlagte häufig deutlich mehr Steuern als tariflich vorgesehen. Im Durchschnitt beträgt die Differenz knapp 119 Euro für alle Nichtveranlagten und 360 Euro, wenn man nur Fälle mit Übereinbehalt berücksichtigt. Insgesamt haben Nichtveranlagte 2014 mindestens 949 Mio. Euro Steuern zu viel entrichtet.

Aus mehreren Gründen ist dies nur eine Untergrenze für das gesamte Steuererstattungspotenzial bei Abgabe einer Steuererklärung. Erstens berücksichtigen wir nur Pauschbeträge für Werbungskosten und Sonderausgaben, während Nichtveranlagte durchaus höhere erstattungsfähige Ausgaben haben können. Zweitens abstrahieren wir von Kirchensteuer und Solidaritätsbeitrag, da diese auf Basis der verfügbaren Daten nicht eindeutig bestimmt werden können. ${ }^{3}$ Drittens berücksichtigen wir für verheiratete Nichtveranlagte mit Steuerklasse IV den Individualtarif, wobei eine gemeinsame Veranlagung in vielen Fällen noch zu-

3 Für beides werden Kinderfreibeträge in der Berechnung berücksichtigt, die für Nichtveranlagte in der Lohn- und Einkommensteuerstatistik nicht erfasst sind. Somit kann insbesondere auch die Rolle der Kirchensteuer als Sonderausgabe nicht modelliert werden.

\section{Abbildung 2 \\ Durchschnittssteuersatz über die \\ Einkommensverteilung \\ mit 95\% Konfidenzintervallen}

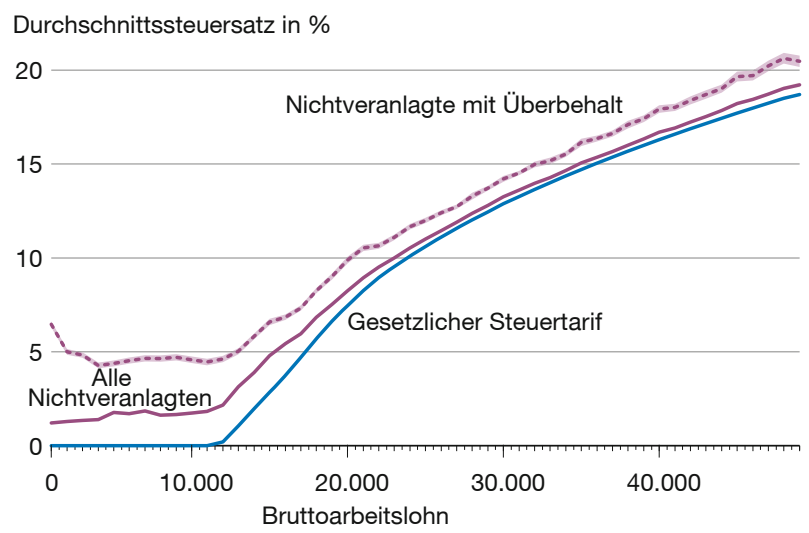

Quelle: Forschungsdatenzentrum des Bundes und der Länder (2020); eigene Berechnungen.

sätzliches Erstattungspotenzial bietet. Viertens haben wir Beamt:innen und Steuerpflichtige jenseits der Beitragsbemessungsgrenze nicht berücksichtigt, jedoch können auch diese Gruppen zu viel Lohnsteuer entrichten. Fünftens sind etwaige Kapitalerträge, die trotz eines nicht vollständig ausgeschöpften Freistellungsauftrags unterjährig besteuert wurden und somit erstattungsfähig sind, nicht erfasst.

\section{Effektive Besteuerung}

Dass Nichtveranlagte mehr Steuern zahlen als im Steuertarif vorgesehen, wirkt sich deutlich auf die effektive Besteuerung aus. Abbildung 2 vergleicht die effektiven Durchschnittssteuersätze mit den tariflich vorgesehenen. Für alle Einkommensniveaus sind die effektiven Steuersätze höher als die tariflichen, die Differenz ist jedoch am unteren Ende der Einkommensverteilung besonders groß.

Der Steuertarif sieht mit dem Grundfreibetrag einen Durchschnittssteuersatz von $0 \%$ für jährliche Bruttoeinkommen bis etwa 11.000 Euro vor. Tatsächlich beträgt der effektive Steuersatz für Nichtveranlagte hier aber etwa $2 \%$. Für Nichtveranlagte mit Steuererstattungspotenzial aus Übereinbehalt beträgt die Abweichung im Mittel effektiv etwa 5 Prozentpunkte in diesem Einkommensbereich.

Das System der freiwilligen Steuererklärungen führt also dazu, dass vor allem Geringverdienende effektiv mehr Steuern zahlen als das Einkommensteuergesetz für ihr Einkommen definiert. Als Resultat ist die effektive Einkommensbesteuerung weniger progressiv als es der Steuertarif vorsieht. 


\section{Handlungsempfehlungen}

Wenn der Steuertarif die gewünschte Progression abbildet, ist die übermäßige Besteuerung Nichtveranlagter ein unerwünschter Nebeneffekt der optionalen Veranlagung. Aus verteilungspolitischer, aber auch aus juristischer Perspektive, ist insbesondere die effektive Besteuerung am unteren Ende der Einkommensverteilung von Relevanz. In seinem Urteil aus dem Jahr 1992 hat das Bundesverfassungsgericht die Einkommensbesteuerung unterhalb eines existenzsichernden Grundfreibetrags für verfassungswidrig erklärt (Bundesverfassungsgericht, 1992). Wie dieser Beitrag zeigt, führt das aktuelle System der freiwilligen Steuererklärungen jedoch zu einer Verletzung dieser Maxime. Nach unseren Berechnungen bezahlen mehr als 1 Mio. nichtveranlagte Steuerpflichtige unterhalb des Grundfreibetrags Lohnsteuer (Hauck und Wallossek, 2021).

Die naheliegende Empfehlung, um die tatsächliche Besteuerung wieder mit der gesetzlich vorgesehenen in Einklang zu bringen, ist eine automatische Rückerstattung. Bereits heute dürften die Finanzverwaltungen der Länder über notwendige Informationen zu soziodemografischen Charakteristika und Einkommen verfügen, um abhängig Beschäftigten vollautomatisiert die zu viel entrichteten Steuern erstatten zu können. Dies entlastet zum einen die aktuell nichtveranlagten Steuerzahlenden, die dadurch im Durchschnitt 119 Euro zurückerstattet bekommen. Zum anderen entlastet dies auch die freiwillig Veranlagten, die eine Steuererklärung abgeben, ohne Ausgaben jenseits der Pauschbeträge geltend zu machen, da sie dadurch Zeit und Aufwand der Steuererklärung sparen. ${ }^{4}$

Für den Fall, dass automatisierte Erstattungen juristisch oder verwaltungstechnisch nicht möglich sind, können die Finanzverwaltungen abhängig Beschäftigte über ihren potenziellen Steuermehreinbehalt informieren. Es ist da-

4 In einem ähnlichen Kontext schlägt Benzarti (2021) eine Vereinfachung des US-amerikanischen Steuersystems vor, um Befolgungskosten zu reduzieren. von auszugehen, dass Nichtveranlagte sich des Übereinbehalts häufig nicht bewusst sind. Übersichtliche und klar strukturierte Informationen zum Steuermehreinbehalt bauen solche Informationsbarrieren ab und erleichtern zudem potenziell die Abgabe einer Steuererklärung.

Unser Beitrag zeigt, dass das System der optionalen Veranlagung mit freiwilligen Steuererklärungen insbesondere Geringverdienenden finanziell schadet, da diese selten eine Steuererklärung abgeben und zeitgleich überproportional häufig zu viel Lohnsteuer entrichten. Aufgrund einer hohen Konsumquote ist die tatsächliche Steuerbelastung dieser Einkommensgruppen bereits ohne die von uns aufgezeigte Verzerrung hoch. Nicht zuletzt deshalb sollten die Einkommensbesteuerung reformiert und automatische Rückerstattungen initiiert werden, um niedrige Einkommen zu entlasten.

\section{Literatur}

Benzarti, Y. (2021), Estimating the Costs of Filing Tax Returns and the Potential Savings from Policies Aimed at Reducing These Costs, Tax Policy and the Economy, (35), 55-85, https://doi.org/10.1086/713493.

Bundesministerium der Finanzen (2019), Datensammlung zur Steuerpolitik Ausgabe 2019, https://www.bundesfinanzministerium.de/Content/DE/ Downloads/Broschueren_Bestellservice/2020-01-30-datensammlungzur-steuerpolitik-2019.pdf (16. November 2021).

Bundesverfassungsgericht (1992), Beschluss des Zweiten Senats vom 25. September 1992 - 2 BvL 5/91, 2 BvL 14/91, 2 BvL 8/91 - Rn. (1 - 97), http://www.bverfg.de/e/ls19920925_2bvl000591.html (10. November 2021).

Forschungsdatenzentrum des Bundes und der Länder (2018), Lohn- und Einkommenssteuerstatistik 2010, Scientific Use File, https://doi.org/10 21242/73111.2010.00.00.3.1.0 (10. November 2021).

Forschungsdatenzentrum des Bundes und der Länder (2020), Lohn- und Einkommenssteuerstatistik 2014, Scientific Use File, https://doi.org/10. 21242/73111.2014.00.00.3.1.0 (10. November 2021).

Gale, W. J. und J. Holtzblatt (1997), On the possibility of a no-return tax system, National Tax Journal, 50(3), 475-485.

Hauck, T. und L. Wallossek (2021), Optional (Non-)Filing and Effective Taxation, https://doi.org/10.5282/UBM/EPUB.77429 (10. November 2021).

US Department of the Treasury (2003), Return-Free Tax Systems: Tax Simplification Is a Prerequisite, Report to the Congress.

Title: Automatic Income Tax Refunds to Relieve Low Incomes

Abstract: Germany is one of more than 30 countries worldwide where employees are exempt from the obligation of filing a tax return. While this optional assessment relieves one of the burden of filing a return, it has individual and distributional costs because non-filers often pay too much income tax. This excess withholding leads to considerable deviations of the effective tax burden from the tax schedule, especially in low-income brackets and particularly for employees with gross wages below the basic allowance. The resulting additional tax revenue amounted to at least 949 million euro in 2014 alone. An automatic refund of this excess withholding would especially relieve low incomes. JEL Classification: H21, H24 